\title{
Brain natriuretic peptide and N-terminal pro-brain natriuretic peptide confirmed the prognostic accuracy in pediatric cardiac surgery: Time for their inclusion in prediction risk models?
}

\author{
Massimiliano Cantinotti, MD, ${ }^{\mathrm{a}, \mathrm{b}}$ and Aldo Clerico, $\mathrm{MD}, \mathrm{PhD}^{\mathrm{a}, \mathrm{c}}$
}

\footnotetext{
From the ${ }^{a}$ Fondazione G. Monasterio CNR-Regione Toscana, Massa; ${ }^{\mathrm{b}}$ Institute of Clinical Physiology; and ${ }^{\mathrm{c}} \mathrm{St}$ 'Anna University, Pisa, Italy.

Disclosures: Authors have nothing to disclose with regard to commercial support.

Received for publication Feb 14, 2017; revisions received Feb 28, 2017; accepted for publication March 13, 2017 available ahead of print April 19, 2017.

Address for reprints: Massimiliano Cantinotti, MD, Fondazione G. Monasterio CNR-Regione Toscana, Ospedale del Cuore, via Aurelia Sud, 54100 Massa, Italy (E-mail: cantinotti@ftgm.it).

J Thorac Cardiovasc Surg 2017;154:641-3

$0022-5223 / \$ 36.00$

Copyright @ 2017 by The American Association for Thoracic Surgery

http://dx.doi.org/10.1016/j.jtcvs.2017.03.022
}

In their article entitled "Perioperative NT-proBNP level: Potential prognostic markers in children undergoing congenital heart disease surgery," Qu and colleagues ${ }^{1}$ evaluated the prognostic accuracy of N-terminal pro-brain natriuretic peptide (NT-proBNP) in 363 consecutive children undergoing cardiac surgery for congenital heart disease. $\mathrm{Qu}$ and colleagues $^{1}$ reported that NT-proBNP values (particularly those collected at 1-hour postsurgery) were significantly associated with a series of outcome markers, including prolonged duration of mechanical ventilation, intensive care unit stay, and inotropic therapy. In particular, a $1 \%$ increase in NT-proBNP values was strongly $(P<.001)$ associated with $5.5 \%$ and $3.9 \%$ relative increases in expected duration of mechanical ventilation and intensive care unit stay, respectively. In particular, NT-proBNP and Risk Adjustment in Congenital Heart Surgery, version 1 scores were found to be independent predictors of prolonged duration of inotropic therapy even after adjusting for confounding variables. NT-proBNP, measured in samples collected at 1 hour after surgery, showed the best accuracy in predicting longer duration of mechanical ventilation (area under the curve, 0.84, with sensitivity of $75.3 \%$ and specificity of $80.4 \%$ at a brain natriuretic peptide [BNP] cutoff value of $2124 \mathrm{pg} / \mathrm{mL}$ ).

The clinical reliance on prognostic biomarkers of B-type cardiac natriuretic peptides (including BNP and NTproBNP) and other biomarkers (eg, thyroid hormones, thyroid-stimulating hormone, and urinary neutrophil gelatinase-associated lipocalin) ${ }^{2-5}$ was reported in previous studies using different sample timing and statistical approach. Among 587 children undergoing cardiac surgery (median age, 6.3 months; 1.2-35.9 months), our group reported that postoperative BNP peak values (with a mean peak occurring at 63.65 hours) were the best predictor for a composite outcome, including major complications and intubation time more than 15 days, improving both hazard ratio and discrimination power compared with other BNP

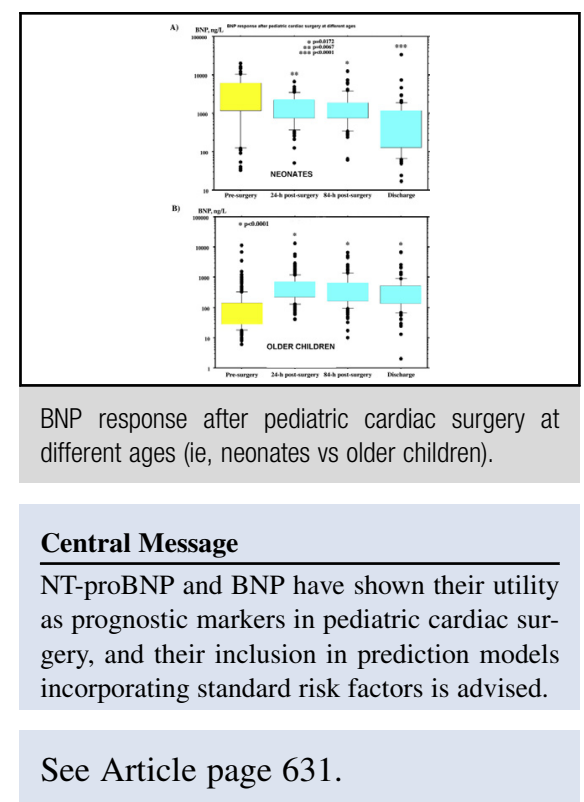

sample values. These data indicate that the use of BNP peak values (instead of BNP values at specified time sampling) may allow a more accurate estimation of hemodynamic balance, because plasma samples collected at 1 hour after cardiopulmonary bypass plasma may be diluted.

Future studies will need to focus attention on 2 areas: the statistical methods and the age categories of the subjects. According to the latest recommendations, ${ }^{6}$ prognostic performance of a novel biomarker should be established by means a complete battery of statistical tests, evaluating calibration, discrimination, and reclassification power.

In addition, it is essential to consider that different trends in BNP values may be observed among neonates and older children. ${ }^{2-4}$ Indeed, although BNP values abruptly decrease after surgery in neonates, older children may show a diphasic pattern with an increase some hours or days after surgery with a progressive decrease thereafter ${ }^{2-4}$ (Figure 1).

The article by $\mathrm{Qu}$ and colleagues ${ }^{1}$ adds new evidence to support the routine use of BNP/NT-proBNP as a prognostic marker in pediatric cardiac surgery. For a more correct clinical interpretation of BNP/NT-proBNP values after cardiac surgery, we recommend that serial blood samples should be evaluated before and after surgery, and trends rather absolute values should be considered. 


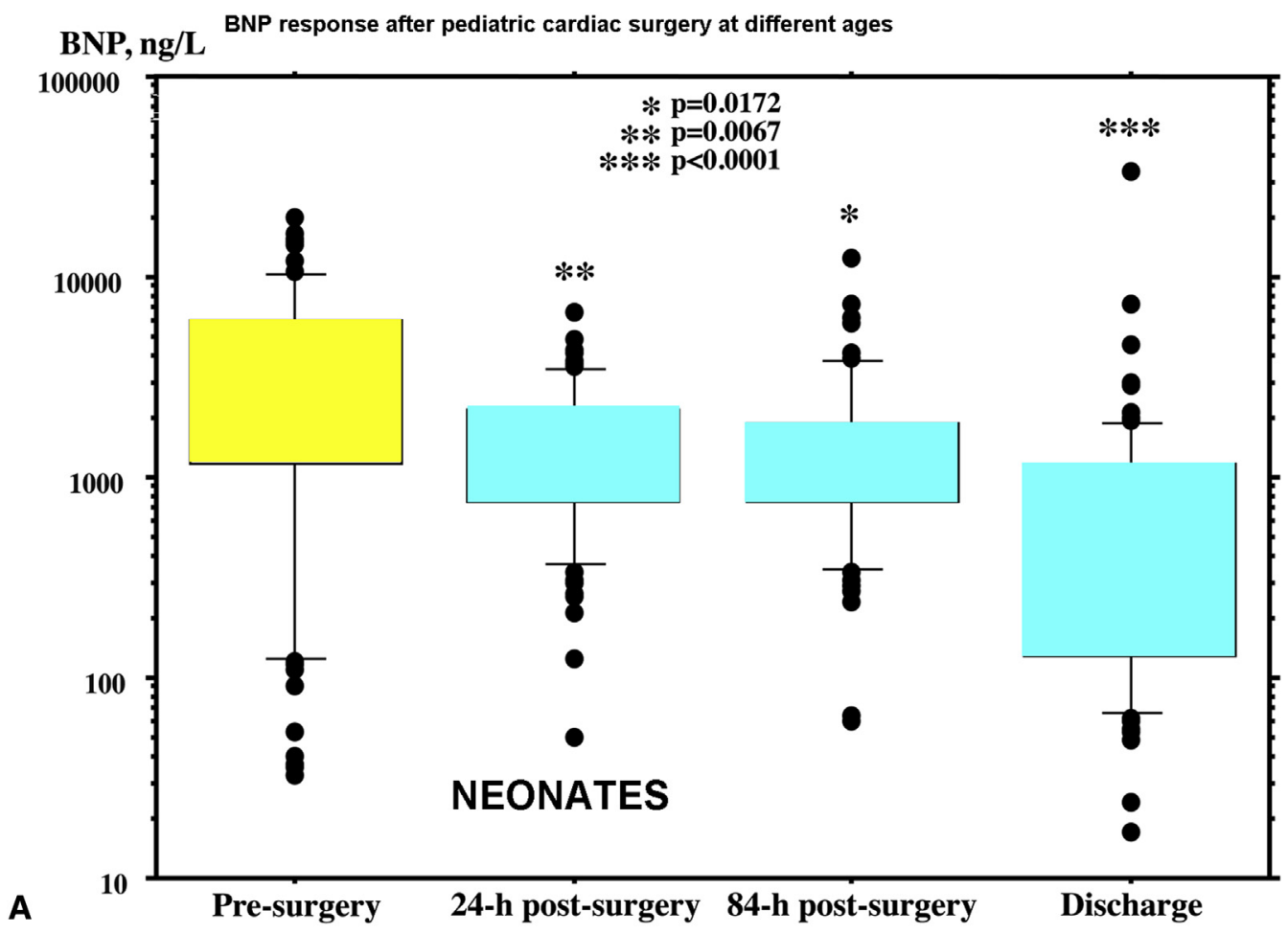

BNP, ng/L

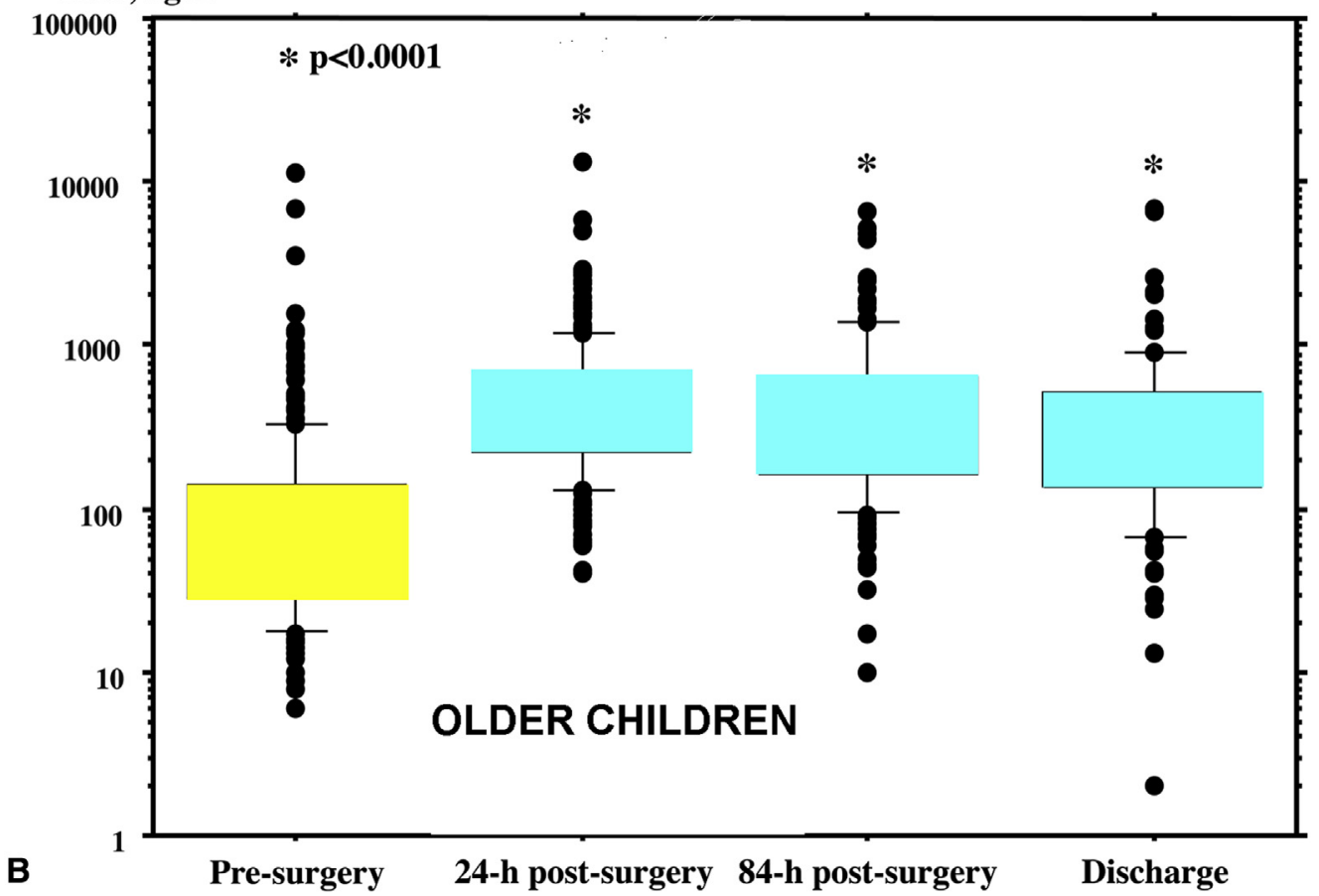

FIGURE 1. Brain natriuretic peptide $(B N P)$ response after pediatric cardiac surgery at different ages (ie, neonates $[\mathrm{A}]$ vs older children [B]).

\section{References}

1. Qu J, Liang H, Zhou N, Li L, Wang Y, Li J, et al. Perioperative NT-proBNP level: potential prognostic markers in children undergoing congenital heart disease surgery. J Thorac Cardiovasc Surg. 2017;154:631-40.

2. Cantinotti M, Lorenzoni V, Storti S, Moschetti R, Murzi B, Marotta M, et al. Thyroid and brain natriuretic peptide response in children undergoing cardiac surgery for congenital heart disease- age-related variations and prognostic value. Circ J. 2013:77:188-97.

3. Cantinotti M, Storti S, Lorenzoni V, Arcieri L, Moschetti R, Murzi B, et al. The combined use of neutrophil gelatinase-associated lipocalin and brain natriuretic peptide improves risk stratification in pediatric cardiac surgery. Clin Chem Lab Med. 2012;50:2009-17. 
4. Cantinotti M, Giordano R, Scalese M, Molinaro S, Della Pina F, Storti S, et al. Prognostic role of BNP in children undergoing surgery for congenital heart disease: analysis of prediction models incorporating standard risk factors. Clin Chem Lab Med. 2015;53:1839-46.

5. Cantinotti M, Vittorini S, Storti S, Prontera C, Murzi M, De Lucia V, et al. Diagnostic accuracy and clinical relevance of brain natriuretic peptide assay in pediat- ric patients with congenital heart diseases. J Cardiovasc Med (Hagerstown). 2009; 10:706-13.

6. Hlatky MA, Greenland P, Arnett DK, Ballantyne CM, Criqui MH, Elkind MS, et al. Criteria for evaluation of novel markers of cardiovascular risk: a scientific statement from the American Heart Association. Circulation. 2009;119: 2408-16. 